\title{
Calibration System of AMiBA Project
}

\author{
K.-Y. Lin ${ }^{1,2}$, D. Kubo ${ }^{2}$, M.-T. Chen ${ }^{2}$, T.-H. Chiueh ${ }^{1}$ \\ ${ }^{1}$ Institute of Physics, National Taiwan University, 1 Roosevelt Rd. Sec. 4, Taipei 106, Taiwan \\ ${ }^{2}$ Institute of Astronomy and Astrophysics, Academia Sinica, 1 Roosevelt Rd. Sec. 4, Taipei 106, Taiwan \\ e-mail: kylin@asiaa.sinica.edu.tw
}

\begin{abstract}
AMiBA is a $3 \mathrm{~mm}$ wavelength astronomical interferometer consisting of $0.6 \mathrm{~m}$ to $1.2 \mathrm{~m}$ antennae. An artificial source is required for its calibration. We present an approach of using a tunable $\mathrm{CW}$ source to calibrate the phase and amplitude response. Performance and problems of the system are discussed.
\end{abstract}

\section{Introduction}

Array for Microwave Background Anisotropy (AMiBA) [1][2] is an interferometer aiming to determine the cosmological parameters, mainly the matter density $\Omega_{\mathrm{m}}$ and the density perturbation normalization $\sigma_{8}$, by measuring the anisotropy in the Cosmic Microwave Background (CMB) radiation at arcminute scale. Though the measurement on CMB angular power spectrum can probe the early universe, its main science target at probing the more recent large-scale structures, i.e. galaxy clusters, through the Sunyaev-Zel'dovich (SZ) effect [3], which results from the distortion in CMB radiation's frequency spectrum due to the inverse Compton scattering by hot electrons contained within galaxy clusters.

AMiBA operates at $86-102 \mathrm{GHz}$ and will eventually consist of 19 antennae / 171 baselines. The array sits on a single platform and the longest baseline will be $6 \mathrm{~m}$, yielding the highest angular resolution 2 arcmin. While a set of $0.6 \mathrm{~m}$ diameter antennae were designed to suit the initial CMB angular power spectrum measurement, another set of $1.2 \mathrm{~m}$ antennae will be in use to optimize collection of the galaxy cluster SZ effect signal. Correlation of each baseline currently takes place in a wide-band 4-lag analog correlator [4][5] at $2-18 \mathrm{GHz}$ intermediate frequency (IF).

A common way of calibration is to observe a strong point source and calibrate out the instrument-introduced phase and amplitude errors. However, point sources that are sufficiently strong in $3 \mathrm{~mm}$, for our relatively small antenna size $0.6 \mathrm{~m}$ or $1.2 \mathrm{~m}$, are rare. An artificial calibrator is therefore required to calibrate the phase and amplitude response of the receiver system frequently.

\section{Artificial Calibrator}

A straight forward way to investigate the system response is to send a CW signal that sweeps through the entire passband and determines the spectral response both in amplitude and in phase. A block diagram of the system can be found in Fig. 1.

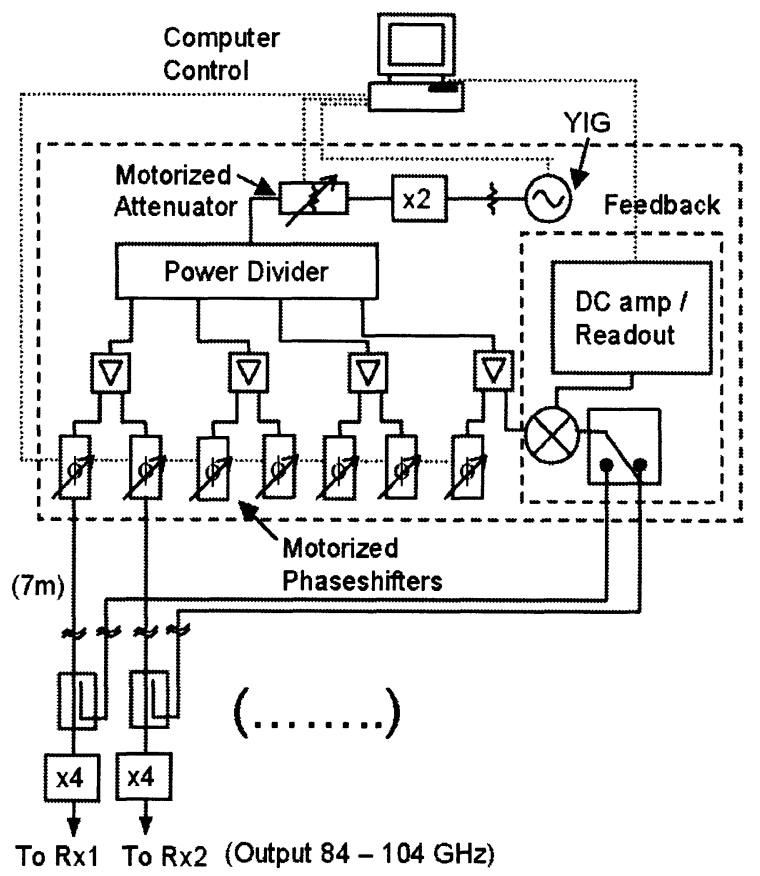

Fig. 1: AMiBA calibration system block diagram. The two dashed boxes 'Feedback' and 'Broadband Noise' were not included in the test result shown in Fig.3. The YTO output $10.5-13 \mathrm{GHz}$ tunable $\mathrm{CW}$ signal. The signal is doubled and distributed to each receiver. An independent phaseshifter is introduced in each path to create relative phase difference. Finally the signal is multiplied by four (x4) and coupled into receiver for correlation.

The 4-lag analog correlator contains four mixers, which have relative delays of $(-37.5,-12.5,12.5,37.5)$ picosec respectively in an ideal case. During calibration, output of a particular mixer can be written as

$$
\begin{aligned}
L\left(f ; \tau_{i}\right)=D_{i} & +P(f) R(f ; i) \\
& \times \cos \left(\phi_{s y s}+\phi_{c a l}+2 \pi\left(f-f_{L o}\right) \tau_{i}\right) .
\end{aligned}
$$

$P(f)$ represents the input $\mathrm{CW}$ power. $R(f ; i)$ indicates the system amplitude response as a function of frequency and mixer variation. Apart from the system phase response $\phi_{s y s}$, the output is also affected by calibration system path difference $\phi_{c a l}$, actual IF delay $\tau_{i}$, and the system DC offset $D_{i}$. 
In order to break the degeneracy, we introduce a variable phase shifter in each calibration signal path as shown in Fig. 1. A full cycle of fringe in each baseline is sampled at one frequency and repeated over the entire frequency band. Fitting a sinusoidal function to data at one frequency determines $R(f ; i), D_{i}$, and $\phi_{c a l}+\phi_{s y s}+2 \pi \tau_{i}(f-$ $\left.f_{L O}\right)=\phi_{c a l}+\phi_{s y s}$ '. $\phi_{s y s}$ and $i$ need not be distinguished, since $\phi_{\text {sys }}$ gives as good description of the system response. To single out $\phi_{c a l}$, it requires an addition astronomical source, such as Jupiter, which calibrates $\phi_{s y s}$ directly. It then combines with the artificial calibrator data to determine $\phi_{c a l}$.

As a calibrator, its $\phi_{c a l}$ and $P(f)$ should be stable, or the variation be known. To achieve the later goal, we include a feedback system to monitor the calibrator variation (Fig. 1). In particular, we insert a coupler at the end of distribution and couple out a weak signal. The signal is sent back to the central source and mixed with a signal before distribution. This allows for monitoring the phase variation of the calibrator.

\section{Applying Calibration Data}

The calibration data $R(f ; i)$ and $\phi_{s y s}$ ' can be thought of as an array of complex response $C\left(i, f_{j}\right)$, or simply $C_{i j}$. With a broad band signal, $P\left(f_{j}\right)$ or $P_{j}$, coming through, the mixer output in matrix representation is

$L_{i}=C_{i j} P_{j} \cdot \Delta f$.

By trying to find an inverse matrix $C_{i j}{ }^{-1}[3]$, one can use the calibration information for transform mixer output data back into complex power spectrum as in complex visibility [6]

$P_{j}=\left(C_{i j}\right)^{-1} L_{i} / \Delta f$.

In AMiBA's case, $i$ runs from 1 to 4 , whereas one can sample arbitrarily number in frequency points. The inversion of matrix $\mathrm{Cij}$ is therefore not unique with infinitely many degenerate solutions. The choice of solution is an optimization problem.

\section{Initial Test Result}

Initial test at three frequencies of the system is shown in Fig. 2. As explained earlier, the relative phase in vertical axis consists of $\phi_{c a l}+\phi_{s y s}+2 \pi \tau_{i}\left(f-f_{L O}\right)$. The solid lines indicate the ideal contribution of $2 \pi \tau_{i}\left(f-f_{L O}\right)$, to which the measured data were quite consistent. The displacement of points from the lines is a measure of $\phi_{c a l}+\phi_{s y s}$. Further tests are required to test other aspects of the system's function.

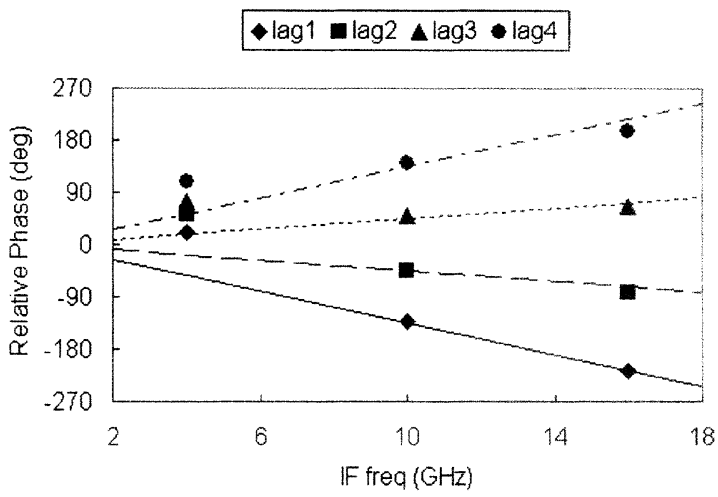

Fig. 2: $\mathrm{CW}$ calibration source test at three different input frequencies 100,94 and $88 \mathrm{GHz}$, corresponding to IF frequencies 16,10 and $4 \mathrm{GHz}$ respectively. Horizontal axis shows the passband frequency range. Vertical axis shows the relative phase between two receivers. Points are measured results. Lines represent the ideal case for each lag.

\section{Known Issues and Plans}

The calibration source is distributed from a common source on the platform to each receiver. Unlike an astronomical source, it is not capable of detecting the phase error induced by the platform deformation. Such phase error must be measured or modeled by other methods. Efforts have been directed to measuring the deformation and eventually compensating the error automatically.

Another issue concerns the stability of the calibration system. The feedback signal is coupled from before the final harmonic generator. The variation in the harmonic generator is not monitored and hence efforts should be made to minimize this. We plan to investigate how severe the variation is and the potential improvement effect of thermal insulation of the device.

We plan to have a fully working 2-element system by September 2005 and a complete system at beginning of 2006.

\section{References}

[1] K.-Y. Lo et al., "AMiBA: Array for Microwave Background Anistropy ", AIPC, vol. 586, 2001, 172L.

[2] Y.-J. Hwang et al., "W-band dual-polarization receiver for array of microwave background anisotropy (AMiBA)", SPIE, vol 5498, 2004, 517H.

[3] R. A. Sunyaev \& Ya. B. Zel'dovich, "Distortions of the Background Radiation Spectrum", Nature, vol. 223, 1969, 721S.

[4] A. I. Harris \& J. Zmuidzinas, "A wideband lag correlator for heterodyne spectroscopy of broad astronomical and atmospheric spectral lines", Review. of Scientific Instrument, vol. 72, 2001, 1531.

[5] C.-T. Li et al., "A wideband analog correlator system for AMiBA", SPIE, vol 5498, 2004, 445L.

[6] A. R. Thompson et al., "Interferometry and Synthesis in Radio Astronomy", John Wiley \& Sons, Inc., $2^{\text {nd }}$ Ed., 2001. 